\title{
Morse simulation of the Global Monopole equation in flat spacetime
}

\author{
S. Habib Mazharimousavi $\left.\right|^{*}$ and M. Halilsoy ${ }^{\dagger}$ \\ Department of Physics, Eastern Mediterranean University, Gazimag̃usa, Turkey.
}

(Dated: October 12, 2018)

\begin{abstract}
We show that a Morse type potential simulates an analytic solution for the highly non-linear global monopole field equation in three and higher dimensional flat spacetimes. Owing to the fact that in the flat space limit the similar equation remains intact we wish to borrow the curved space terminology of global monopole also in flat spacetime. This may provide a compelling example that can be used effectively in different non-linear theories such as flat space $\phi^{4}$, as well as in curved spacetimes.
\end{abstract}

Keywords: Classical field theory; Flat spacetime; Global monopole; Morse potential;

\section{INTRODUCTION}

In the absence of exact solutions non-linear differential equations of field theory manifest features that they can be simulated by some known potentials. Linear equations with complicated potentials also can be treated similarly. In this regard we recall the $H$-atom endowed with non-linear electromagnetic field due to Born and Infeld 1]. In this approach basic idea was to eliminate the divergences due to point charges. The resulting regular electromagnetic potential expected to contribute to the solution of Schrödinger equation and the ground state energy level was obtained remarkably as a simulation [2] of the Morse potential [3]. Being motivated by that study we wish to employ the same method to another well-known differential equation representing an analog of global monopole in flat spacetime. Global monopoles are topological structures that emerge from spontaneous symmetry breaking and are believed to exist from the big bang. Such a monopole was considered first by Barriola and Vilenkin 44 as source of gravity which may go to the extend of forming black holes. In $3+1$-dimensions the symmetry that has been broken is $O(3)$ which ultimately reduces to $U(1)$. In this approach [4] the scalar field triplet is given by $\phi^{a}=\eta f(r) \frac{x^{a}}{r}$, where $a=1,2,3$ are the gauge parameters, $\eta$ is the monopole charge and $f(r)$ is the function to be determined. Unfortunately the differential equation satisfied by $f(r)$ doesn't admit an exact analytical solution. The asymptotic behaviors satisfied by $f(r)$ are such that $f(r) \rightarrow 0$ for $r \rightarrow 0$, and $f(r) \rightarrow 1$ with $f^{\prime}(r)=0$ for $r \rightarrow \infty$. Numerical integration of the resulting differential equation is at our disposal and our aim is to simulate such a global monopole equation by using a Morse type potential. Before extending our formalism to higher dimensions we consider the 2+1-dimensional analogue of the $3+1$-dimensional global monopole. In $2+1$-dimensions the gauge group to be broken becomes now $O(2)$ so that the scalar doublet is represented by $\phi^{a}=\eta f(r) \frac{x^{a}}{r}$, where $a=1,2$. The resulting differential equation for $f(r)$ in $2+1$-dimensions

\footnotetext{
* habib.mazhari@emu.edu.tr

$\dagger$ mustafa.halilsoy@emu.edu.tr
}

is as difficult as in the $3+1$-dimensional case. As a matter of fact asymptotically the resulting equation is reminiscent of a minor variation of the Third Painlevé transcendent [5] encountered in the study of cylindrical gravitational waves [6]. This oscillatory solution, however, doesn't represent the monopole character but yet arises as a distinct solution to the same differential equation satisfied by the monopole. In other words both types of solutions arise from the same differential equation with different initial conditions. Morse potential fitting to the global monopole equation in $2+1$-dimensions is observed to work perfectly. Going to the lower dimension $1+1$, the differential equation satisfied by $f(r)$ is solved exactly as $f(r)=\tanh \left(\frac{r}{\sqrt{2}}\right)$. This is, however, a singlet field and it can't be interpreted as a global monopole since there is no symmetry breaking in the usual sense. Reflection symmetry is the only available symmetry to be in 1-dimensional space. Next, we interpolate our analysis to higher dimensions $D \geq 4$ and repeat our method of Morse simulation. We observe that as $D$ gets higher the fitting of our Morse type potential becomes less reliable in comparison with the dimensions $2+1$ and $3+1$.

\section{GLOBAL MONOPOLE EQUATION IN $2+1$-DIMENSIONS}

Let's start with the Lagrangian of a global-monopole in $2+1$-dimensional flat, spherically symmetric spacetime which is given by

$$
\mathcal{L}^{\text {field }}=-\frac{1}{2} \partial_{\mu} \phi^{a} \partial^{\mu} \phi^{a}-\frac{1}{4} \lambda\left(\phi^{a} \phi^{a}-\eta^{2}\right)^{2} .
$$

Here $a=(1,2), \lambda$ is a coupling constant, $\eta$ is the monopole parameter and

$$
\phi^{a}=\eta f(r) \frac{x^{a}}{r},
$$

for $x^{1}=r \cos \theta$ and $x^{2}=r \sin \theta$. To find the field equation for $f(r)$ we express the field Lagrangian in terms of $f(r)$ only, i.e.,

$$
\mathcal{L}^{\text {field }}=-\frac{\eta^{2}}{2}\left(f^{\prime 2}+\frac{f^{2}}{r^{2}}\right)-\frac{\lambda \eta^{4}}{4}\left(f^{2}-1\right)^{2} .
$$


Now, variation of the action with respect to $f$ yields

$$
\left(r f^{\prime}\right)^{\prime}-\frac{f}{r}-\lambda \eta^{2} r f\left(f^{2}-1\right)=0
$$

or in more convenient form

$$
f^{\prime \prime}+\frac{1}{r} f^{\prime}+\left(\frac{1}{\delta^{2}}-\frac{1}{r^{2}}\right) f=\frac{1}{\delta^{2}} f^{3}
$$

in which a prime stands for the derivative with respect to $r$ and $\delta=\frac{1}{\eta \sqrt{\lambda}}$ is the size of the global monopole in flat spacetime. We note that a scaling of the form $r \rightarrow r \delta$, absorbs the parameter, hence without loss of generality one can set $\delta=1$. Note also that the term $-\frac{f}{r^{2}}$ is as a result of symmetry breaking.

Let us note that Eq. (5) admits an asymptotic solution in analogy with the cylindrical gravitational waves expressed exactly in terms of Painlevé type III [6]. Our case turns out to be

$$
\begin{aligned}
f(r) & \sim \frac{1}{\sqrt{r}} \cos \left(r+\frac{3}{8} \ln r+\alpha_{0}\right)- \\
& \frac{1}{32 r^{3 / 2}} \cos 3\left(r-\frac{3}{8} \ln r+\alpha_{0}\right)+O\left(r^{-5 / 2}\right)
\end{aligned}
$$

(for $\alpha_{0}=$ constant) which is comparable with the asymptotic solution given in [6].

In Fig. 1 we plot the solution of latter equation for $\delta=1$ and $f(0)=0$. The solution is oscillatory for $f^{\prime}(0)<0.583189028596$, asymptotes to one for $f^{\prime}(0)=0.583189028596$ and diverges for $f^{\prime}(0)>$ 0.583189028596 . Interestingly the decisive factor between the two types of solutions is the initial slope of the curve to start with. Below certain value we have oscillations while above that we have the frozen global monopole solution.

In Fig. 2 we plot the numerical solution of Eq. (5) which represents a global monopole with $\delta=1$ and the Morse type function

$$
f(r) \simeq 1-\left(f_{0}^{\prime}-1\right) e^{-2 r}+\left(f_{0}^{\prime}-2\right) e^{-r}
$$

in which $f_{0}^{\prime}=0.583189028596$. Obviously $f(r)$ satisfies the conditions at $r=0$ and $r \rightarrow \infty$.

\section{GLOBAL MONOPOLE EQUATION IN ALL DIMENSIONS}

In higher dimensional spherical symmetric flat spacetime the action of a global monopole field is given by (1)

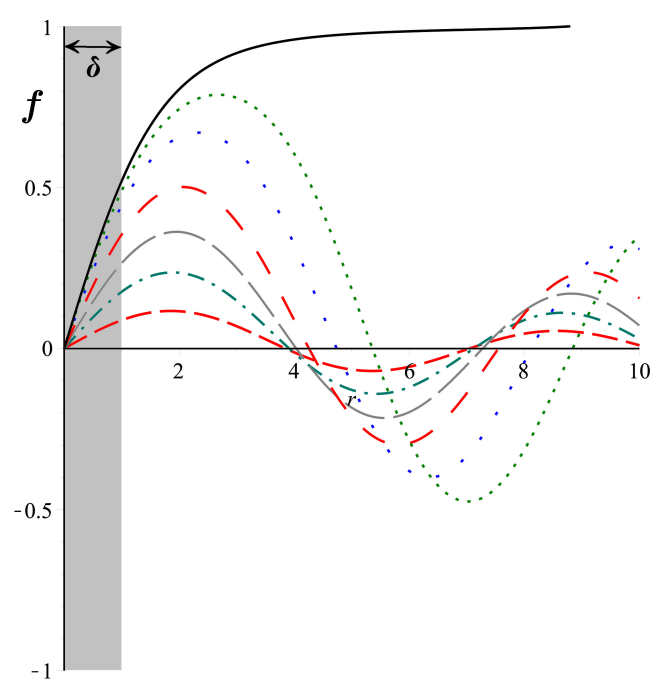

FIG. 1: A plot of the solution to the field equation (5) $f(r)$ with respect to $r$ for $\delta=1$ in flat spacetime. The initial conditions are set as $f(0)=0$ and $f^{\prime}(0)=0.583189029,0.55,0.5,0.4,0.3,0.2$ and 0.1 from the top/Black-Solid to the bottom/Red-long Dash. Note that the shaded region corresponds to the inner region of global-monopole. We add also that depending on the initial slope Eq. (5) admits a wave like solution which is apart from the monopole. For completeness we have shown the latter as well.

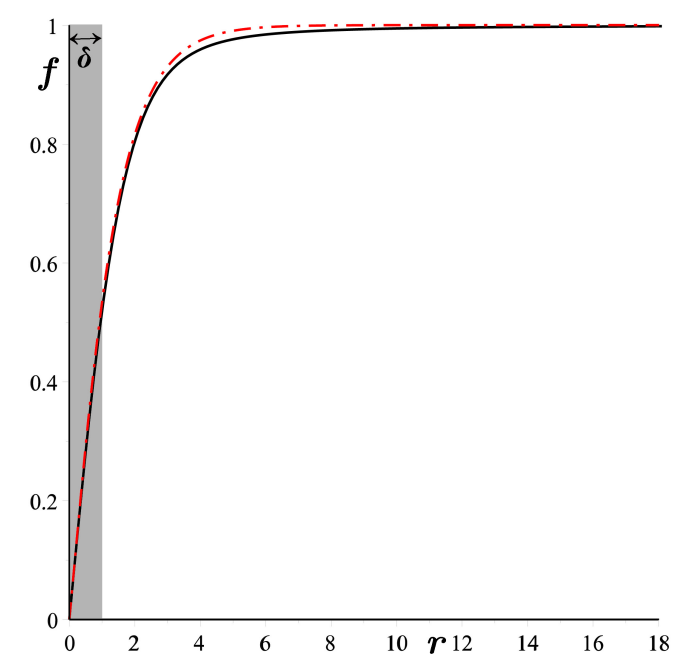

FIG. 2: A plot (black-solid) of the solution to the field equation (5) $f(r)$ with respect to $r$ for $\delta=1$ in flat spacetime for the initial conditions are set as $f(0)=0$ and $f^{\prime}(0)=0.583189029$, and the Morse function (red-dash). 
and (2), with $a=(1,2, \ldots, D-1)$ and

$$
\begin{aligned}
x^{1}= & r \cos \theta_{1} \\
x^{2}= & r \sin \theta_{1} \cos \theta_{2} \\
x^{3}= & r \sin \theta_{1} \sin \theta_{2} \cos \theta_{3} \\
& \cdot \\
& \cdot \\
& \cdot \\
x^{D-2}= & r \sin \theta_{1} \ldots \sin \theta_{D-3} \cos \theta_{D-2} \\
x^{D-1}= & r \sin \theta_{1} \ldots \sin \theta_{D-3} \sin \theta_{D-2}
\end{aligned}
$$

where $\theta_{D-2} \in[0,2 \pi]$ and $\theta_{k} \in[0, \pi]$ with $k=1,2, \ldots, D-$ 3 . The arbitrary dimensional action can be expressed in the form

$$
\mathcal{L}^{\text {field }}=-\frac{\eta^{2}}{2}\left(f^{\prime 2}+\frac{D_{2} f^{2}}{r^{2}}\right)-\frac{\lambda \eta^{4}}{4}\left(f^{2}-1\right)^{2}
$$

in which $D_{2}=D-2$ with $D$ the dimension of the spacetime. The field equation following the above Lagrangian is found to be

$$
f^{\prime \prime}+\frac{D_{2}}{r} f^{\prime}+\left(\frac{1}{\delta^{2}}-\frac{D_{2}}{r^{2}}\right) f=\frac{1}{\delta^{2}} f^{3}
$$

with $\delta=\frac{1}{\eta \sqrt{\lambda}}$ the size of the higher dimensional global monopole. In Fig. 3 we plot the global monopole solution of the latter equation for $D=2$ (top/black-long dash), $D=3,4,5,6$ and $D=10$ (bottom/purple-dot). The case when the dimension is two i.e., $D=2$ is analytically solvable. The field equation for $D=2$ and $\delta=1$ can be written as

$$
f^{\prime \prime}+f\left(1-f^{2}\right)=0
$$

with a first integral

$$
f^{\prime 2}=\frac{1}{2} f^{4}-f^{2}+C
$$

in which $C$ is an integration constant. As we are looking for a global monopole type solution, we impose the constraint that when $r \rightarrow \infty, f \rightarrow 1$ and $f^{\prime} \rightarrow 0$. This in turn implies that $C=\frac{1}{2}$. Upon considering $C$ in (12) we obtain

$$
f^{\prime 2}=\frac{1}{2}\left(f^{2}-1\right)^{2} .
$$

Furthermore the global monopole field admits a positive derivative and is less than one which imply

$$
f^{\prime}=\frac{1}{\sqrt{2}}\left(1-f^{2}\right)
$$

This final equation admits an exact solution given by

$$
f=\tanh \left(\frac{r}{\sqrt{2}}+\tilde{C}\right)
$$

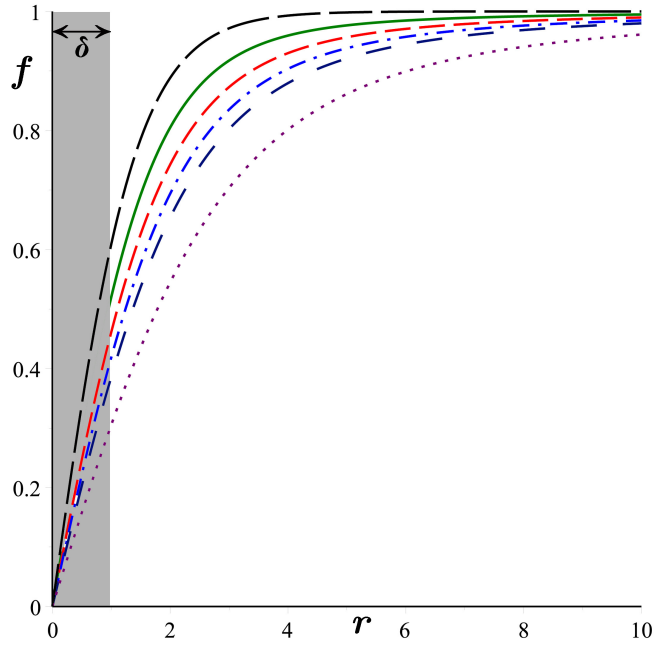

FIG. 3: Plot of numerical global monopole solution to the general field equation (10) for dimensions $D=2,3,4,5,6,10$ from the top to the bottom.

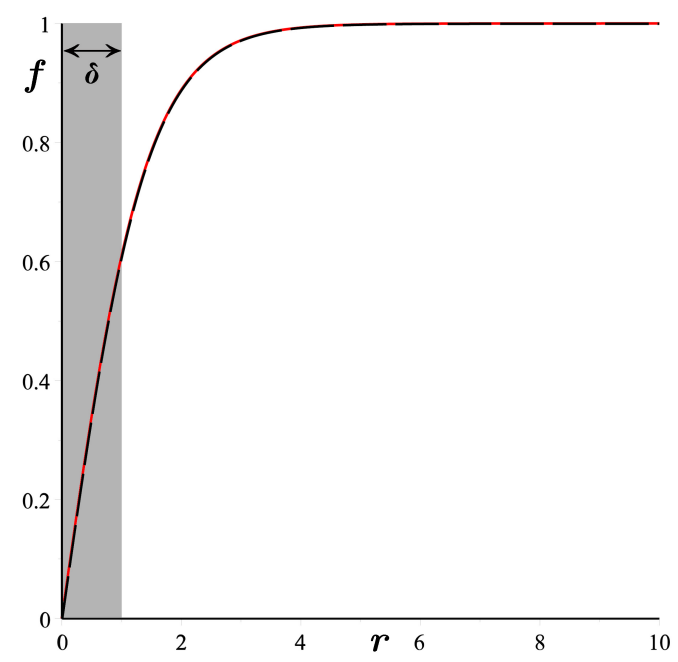

FIG. 4: Plot of exact solution for the global monopole function $f$ and the numeric solution for $D=2$. The two solutions agree highly which can not be distinguished from each other.

in which $\tilde{C}$ is an integration constant. Imposing $f(0)=$ 0 , one finds $\tilde{C}=0$ which makes the final solution simply as

$$
f=\tanh \left(\frac{r}{\sqrt{2}}\right)
$$

In Fig. 4 we plot the exact solution (16) and the numerical solution found from the field equation. We see that they agree to high precision. This accuracy guides our numerical method in $D=3$ also to the higher dimensions.

Similar to $D=3$, for $D \geq 4$ we can simulate the 


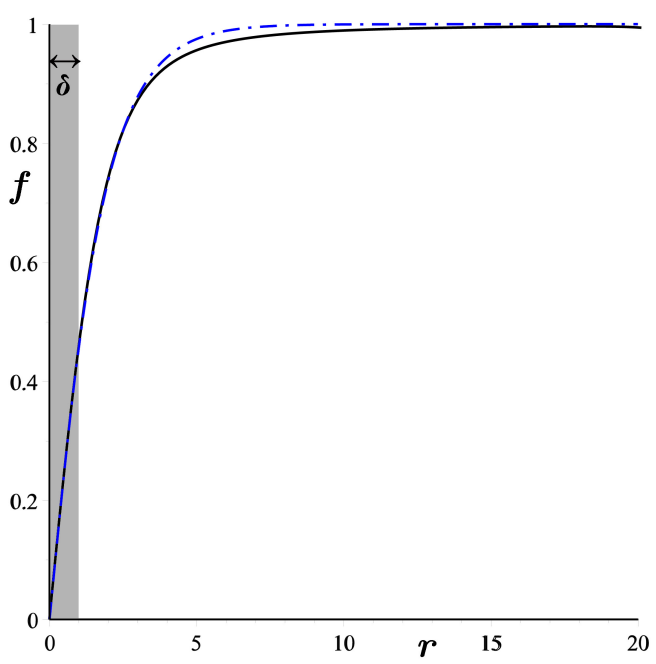

FIG. 5: Plot of simulating Morse function (blue-dash) for the global monopole function $f$ and the numeric solution (black-solid) for $D=4$. In the Morse function we set $\kappa=0.8$. The two solutions agree in both small and large $r$ to high accuracy.

solution with a Morse type function given by

$$
f(r) \simeq 1-\left(\frac{f_{0}^{\prime}}{\kappa}-1\right) e^{-2 \kappa r}+\left(\frac{f_{0}^{\prime}}{\kappa}-2\right) e^{-\kappa r}
$$

in which $f_{0}^{\prime}$ is the slope of the solution at $r=0$ and $\kappa$ is an adjusting parameter. In Table. 1 we give $f_{0}^{\prime}$ for dimensions $D \geq 4$ and in Fig. 5 we plot the numerical solution with the simulated function for $D=4$.

TABLE I: $f_{0}^{\prime}$ for different dimensions.

\begin{tabular}{cc}
\hline \hline$D$ & $f_{0}^{\prime}$ \\
\hline 2 & 0.707106680906 \\
3 & 0.583189028596 \\
4 & 0.506042540468 \\
5 & 0.4525666770699 \\
6 & 0.4128548316012 \\
10 & 0.3188665791567 \\
\hline
\end{tabular}

\section{WHY THE MORSE TYPE FUNCTION?}

Having the solution of Eq. (10) simulated by the Morse type function is not a coincidence. The initial form of the Morse potential can be written as 3 ]

$$
V_{\text {Morse }}(r)=D_{e}\left(1-e^{-a\left(r-r_{e}\right)}\right)^{2}
$$

in which $D_{e}, a$ and $r_{e}$ are free positive parameters of the potential. This function has a minimum at $r=r_{e}$ and for large $r$ it approaches to $D_{e}$. Setting $D_{e}=1$ and $r_{e}=0$ we find a function whose asymptotic behaviours are as we are expecting for $f(r)$ in Eq. (10) i.e., $\lim _{r \rightarrow 0} V_{\text {Morse }}(r)=0$ and $\lim _{r \rightarrow \infty} V_{\text {Morse }}(r)=1$. Furthermore, for large $r$ i.e., $\frac{r}{\delta} \gg 1$, Eq. (10) becomes independent of dimensions and therefore the solution must be asymptotically the same as the exact solution of (10) in $D=2$ given by (16). Hence we get

$$
\lim _{r \rightarrow \infty} f=\lim _{r \rightarrow \infty} \tanh \left(\frac{r}{\zeta}\right)
$$

where $\zeta$ is a positive constant which could be set according to the dimensions. The asymptotic behavior of the right hand side in (19) surprisingly is of the Morse type function i.e., for large $r$

$$
\tanh \left(\frac{r}{\zeta}\right) \sim 1-2 e^{\frac{-2 r}{\zeta}}+2 e^{\frac{-4 r}{\zeta}} .
$$

Finally, for small $r$ i.e., $r / \delta \ll 1$ the field equation (10) becomes

$$
r^{2} f^{\prime \prime}+D_{2} r f^{\prime}-D_{2} f=0
$$

with a solution given by

$$
f(r)=C_{1} r+C_{2} r^{-D_{2}}
$$

in which $C_{1}$ and $C_{2}$ are constants. A regular solution at $r=0$ requires $C_{2}=0$ and the asymptotic solution for small $r$ becomes $f(r)=C_{1} r$. From the other hand the asymptotic behaviour of the Morse potential (18) with $D_{e}=1$ and $r_{e}=0$ near $r=0$ becomes

$$
\lim _{r \rightarrow 0} V_{\text {Morse }}(r) \sim a r
$$

which is same as $f(r)$. In conclusion the Morse type function in both limits i.e., $r \rightarrow 0$ and $r \rightarrow \infty$ matches very well with the solution of the field equation (10). As we expect from the Fig. 5, the maximum agreement occurs in these limits.

\section{CONCLUSION}

Irrespective of dimensions $(D \geq 3)$ the geometry of a global monopole emerges complicated enough to be tackled with, even in a flat spacetime. The reason is the transcendental differential equation satisfied by the global monopole function $f(r)$. Asymptotically the equation satisfied by $f(r)$ has similarity with the Painlevé type III, whose behavior can be studied numerically. We have shown that the numerical plot of the function can be simulated by a Morse type function to great accuracy. It is shown that the accuracy of our simulation works better in lower dimensions such as $D=3,4 . D=2$ is the only available case that admits exact integration, however, its significance as a monopole remains questionable. Recalling that a global monopole constitutes a particular case of $\phi^{4}$ theories similar simulations may find application 
also in the latter theories. Further, question can naturally be raised about the importance of such a simulation advocated herein: the physics of particles in the field of a monopole becomes tractable through the Morse function. Our project next is to extend the idea to global monopole in a curved spacetime.

[5] E. L Ince, "Ordinary Differential Equations", Published by Dover, New York, (1956).

[6] S. Chandrasekhar, Proc. Roy. Soc. A 408, 209 (1986).
[1] M. Born and L. Infeld, Proc. Roy. Soc, A 144, 425 (1934).

[2] S. H. Mazharimousavi and M. Halilsoy, Found Phys. 42, 524 (2012).

[3] P. M. Morse, Phys. Rev. 34, 57 (1929).

[4] M. Barriola and A. Vilenkin, Phys. Rev. Lett. 63, 341 (1989). 